\title{
BMJ Open Cohort profile: The Forzani \& MacPhail Colon Cancer Screening Centre biorepository, Calgary, Alberta
}

\author{
Robert J Hilsden (10 , ${ }^{1}$ Steven J Heitman, ${ }^{1}$ Mubasiru Lamidi, ${ }^{1}$ \\ Mamadou Dian Diallo, ${ }^{1}$ Janine English, ${ }^{1}$ Susanna Town, ${ }^{1}$ Shane Cartwright, ${ }^{1}$ \\ Courtney Maxwell, ${ }^{1}$ Mary-Elizabeth McGillivray, ${ }^{1}$ Elizabeth McGregor, ${ }^{1}$ \\ Amanda Barberio, ${ }^{2}$ Joy Pader, ${ }^{2}$ Darren Brenner (iD , ${ }^{2,3,4}$ Linda Rabeneck ${ }^{5}$
}

To cite: Hilsden RJ, Heitman SJ, Lamidi M, et al. Cohort profile: The Forzani \& MacPhail Colon Cancer Screening Centre biorepository, Calgary, Alberta. BMJ Open 2020;10:e038119. doi:10.1136/ bmjopen-2020-038119

- Prepublication history and supplemental materials for this paper is available online. To view these files, please visit the journal online (http://dx.doi. org/10.1136/bmjopen-2020038119).

Received 27 February 2020 Revised 14 October 2020 Accepted 20 0ctober 2020
Check for updates

(C) Author(s) (or their employer(s)) 2020. Re-use permitted under CC BY-NC. No commercial re-use. See rights and permissions. Published by BMJ.

For numbered affiliations see end of article.

Correspondence to Dr Robert J Hilsden; rhilsden@ucalgary.ca

\section{ABSTRACT}

Purpose The Colon Cancer Screening Centre (CCSC) biorepository (Calgary, Canada) supports a wide range of research topics related to colorectal cancer (CRC) by collecting, and storing biospecimens (blood, urine, normal colon tissue) from consenting patient participants. Housing unique biospecimens along with detailed participant lifestyle and health history questionnaire data, the CCSC biorepository can support a variety of research related to CRC risk factors, biomarkers, genetic causes and more. Participants Currently, 2292 average risk CRC patients have consented to participate in the CCSC cohort and have provided stored biospecimens. The collected samples and data provide important high-quality materials for research, discovery and evaluation related to CRC screening and carcinogenesis and is available for access by outside researchers. In addition to biological samples, the CCSC collects detailed patient information on their lifestyle, physical activity and dietary patterns through questionnaires at the time of their enrolment.

Findings to date The majority of participants $(75 \%)$ are between 50 and 64 years of age. Women make up 46\% (1055) of the cohort. Additional characteristics of the cohort included $44 \%$ reporting a body mass index of $25-$ $30 \mathrm{~kg} / \mathrm{m}^{2}$ (overweight), 53\% having never smoked tobacco and $13 \%$ having a family member with CRC.

Future plans The CCSC cohort plans to include the recruitment of high risk CRC cohorts. High-risk participants would comprise patients with a positive faecal immunochemical test and family history of CRC.

\section{INTRODUCTION}

Colorectal cancer (CRC) is the second most commonly diagnosed cancer in Canada with approximately 26800 new cases diagnosed in 2017. ${ }^{1}$ CRC is the leading cause of cancer-related death among Canadian men and the third leading cancer-related cause of death in Canadian women. ${ }^{1}$ Comparatively, Canada had a 35.8 (95\% CI 35.8 to 41.4$)$ agestandardised incidence rate for both sexes combined (per 100000 person-years) for CRC which were similar to other high income countries such as USA and the UK, with 39.1

\section{Strengths and limitations of this study}

- The Colon Cancer Screening Centre (CCSC) biorepository (Calgary, Canada) supports a wide range of research topics related to colorectal cancer (CRC) by collecting, and storing biospecimens (blood, urine, normal colon tissue) from consenting patient participants.

- From 2008 to 2016, 2292 patients at average risk for CRC have consented to participate in the CCSC cohort and have provided biospecimens prior to undergoing a complete colonoscopy.

- Information on lifestyle, physical activity and dietary patterns was collected at the time of enrolment.

- The main limitation of this cohort is the relatively small population size, and while the questionnaires provide comprehensive information, the questionnaires can only offer cross-sectional data.

(95\% CI 38.0 to 40.4$)$ and 41.7 (95\% CI 40.7 to 42.9), respectively (per 100000 person-years) in 2017. ${ }^{2}$ CRC generally develops following a multistep progression from normal tissue, to hyperproliferation and dysplasia to the formation of an adenomatous polyp and then to cancer. ${ }^{3}$ Known risk factors for CRC include a personal history or family history of CRC and inflammatory bowel diseases. ${ }^{4}$ Additional lifestyle factors such as increased body mass index, ${ }^{6}{ }^{6}$ cigarette smoking, ${ }^{7}$ alcohol consumption, ${ }^{8}$ red meat intake, ${ }^{910}$ low dietary fibre intake ${ }^{11}$ and low physical activity ${ }^{12} 13$ have been shown to increase the risk of CRC. Epigenetic markers including site-specific changes in DNA methylation, and micro RNA expression have also been associated with higher risk of both precancerous adenomas and CRC, although more research on these markers is needed. ${ }^{1415}$

Early detection of CRC through screening asymptomatic individuals reduces the risk of dying from the disease. ${ }^{16}$ Testing of stool for 
occult blood and colonoscopy are the most commonly used screening tests in Canada. ${ }^{17}$ Guidelines from the 2016 Canadian Task Force on Preventive Healthcare recommends screening for CRC in average-risk adults aged 50-74 years with a faecal test, (either the guaiac faecal occult blood test (gFOBT) or faecal immunochemical test (FIT) ) every 2 years, or with flexible sigmoidoscopy every 10 years. ${ }^{18}$ Prior to the updated 2016 guidelines, the CRC screening recommendation for average-risk individuals was a faecal test every 1-2 years or flexible sigmoidoscopy every 5 years starting at age 50 for people at average-risk of CRC. ${ }^{18}$ The current Canadian CRC screening guidelines are aligned with international guidelines from Australia, Denmark, Norway, France, Switzerland and the USA, which also recommends FIT and/or gFOBT for CRC screening among average risk populations starting at age $50 .{ }^{19}$ Facilities devoted to CRC screening provide an opportunity to support research directed at early detection, prevention and treatment of CRC. The Forzani \& MacPhail Colon Cancer Screening Centre located in Calgary, Alberta, is a publically funded facility dedicated to providing CRC screening-related colonoscopies. The CCSC is operated by Alberta Health Services, a provincial health authority that delivers healthcare on behalf of the Alberta Government in partnership with the Faculty of Medicine at the University of Calgary. Those eligible to undergo colonoscopy at the CCSC include those with a positive FIT, those with a personal or family history of CRC or adenomatous polyps and those at average risk for CRC. The CCSC biorepository was created to support research on the development and detection of CRC and other bowel disorders and contains blood, urine and colon tissue samples from consenting research participants. This cohort offers researchers access to a large number of well characterised biological samples combined with detailed lifestyle and family history questionnaire data collected from participants undergoing colonoscopy. The availability of high-quality materials encourages research on lifestyle risk factors, genetic effects, biomarkers, prevention, treatment and evaluation related to CRC and other bowel disorders.

\section{COHORT DESCRIPTION}

The largest cohort (Average Risk Cohort) in the CCSC biorepository represents the target population of CRC screening programmes using non-invasive screening tests, such as FIT. This group includes men or women age 50 years or older at average risk for CRC, without a personal or family history of CRC, or adenomatous polyps, and free of diseases that increases CRC risk, such as inflammatory bowel disease. This group also includes those individuals with a single first degree relative with CRC diagnosed over age 60. To date, the Average Risk Cohort includes 2292 participants recruited from 2008 to 2016 who provided a blood and urine sample within 180 days of their colonoscopy, completed a HLQ and underwent a colonoscopy that was complete to the cecum. To be eligible for recruitment, participants had to be free of significant gastrointestinal symptoms, not to be undergoing a diagnostic colonoscopy due to a positive occult blood test and be due for screening (no colonoscopy within the prior 10 years or flexible sigmoidoscopy or CT colonography within the prior 5 years). They also had to be free of significant medical comorbidities (predominantly ASA class I/II) to meet the CCSC's medical criteria to undergo a colonoscopy under conscious sedation.

Potentially eligible participants were identified from the CCSC's referral database and were sent a study invitation letter by mail. A general diagram of the recruitment process is outlined in figure 1. Characteristics of the CCSC biorepository cohort at enrolment are presented in table 1.

Interested patient participants are mailed an information booklet that provides details on the requirements of the study and a consent form for participation in the study. Participants consent to providing biospecimen samples, questionnaire data, being contacted for follow-ups, and data linkages to health utilisation and outcomes information. Appropriate project-specific ethics authorisation is required for access to participant samples or data.

\section{How often have they been followed up?}

Participants of the CCSC cohort have consented to be regularly contacted to update their health information. Participants receive mailed surveys every 2 years to collect information regarding new diagnoses of CRC, changes to their family's cancer history and completion of additional screening tests and colonoscopies. A transition to online questionnaire completion and recontact processes is currently underway. Additional patient recontact may be possible for approved studies. The CCSC also performs regular linkages with the Alberta Cancer Registry, which has over $99 \%$ coverage of all cancer diagnoses in the province. Any interval cancers or cancers detected outside of the CCSC are included in the biorepository dataset. Individuals who move or die outside of Alberta are considered lost to follow-up.

\section{What has been measured?}

All participants completed a set of questionnaires and provided biospecimen samples prior to undergoing a colonoscopy. The questionnaires collect detailed information on the participant's medical history, lifestyle factors, physical activity and diet (see online supplemental table 1 ). All questionnaires were scanned using TELEform, a data capture software (OpenText, Waterloo, Ontario, Canada; V.8.1-V.16.2) to verify questionnaires and digitalise participant data. Any issues with questionnaire responses from participants were clarified with the participants either at the time the questionnaire was administered or by telephone follow-up. Digital copies of questionnaires were kept after review and cleaning. 


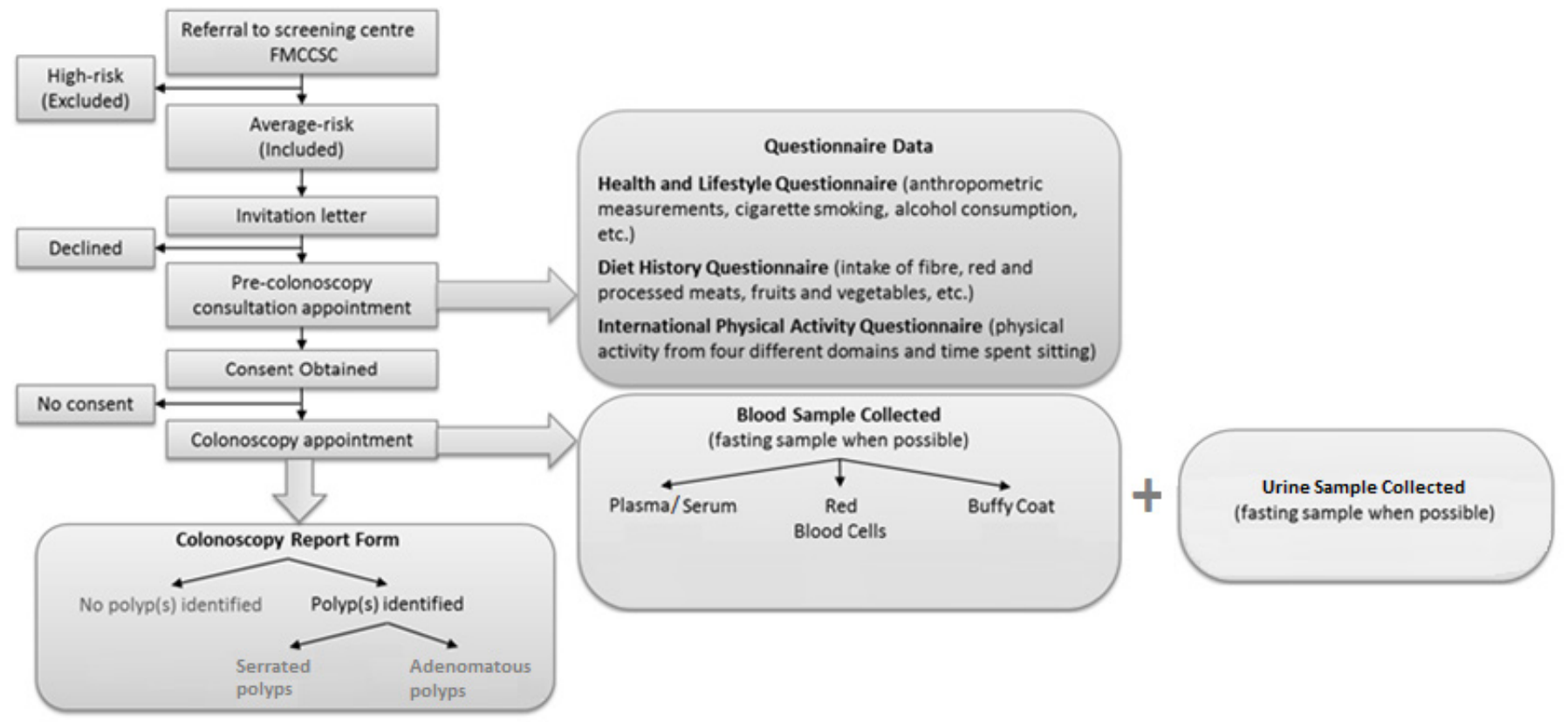

Figure 1 Overview of the CCSC Biorepository recruitment and data collection process. CCSC, Colon Cancer Screening Centre; FMCCSC, Forzani \& MacPhail Colon Cancer Screening Centre.

\section{Health and Lifestyle Questionnaire}

The Health and lifestyle questionnaire (HLQ) collects information on a participant's personal health, family health history, medication use (eg, non-steroidal

\begin{tabular}{lr}
\hline $\begin{array}{l}\text { Table } 1 \\
\text { cohort }\end{array}$ & Characteristics of CCSC biorepository average risk \\
\hline Demographic characteristics & N (\%) \\
\hline $\begin{array}{l}\text { Sex } \\
\text { Male }\end{array}$ & $1237(54)$ \\
\hline Female & $1055(46)$ \\
\hline Age group & \\
\hline $50-64$ & $1729(75)$ \\
\hline $65-75$ & $563(25)$ \\
\hline Country of birth & $1734(75)$ \\
\hline Canada & $540(24)$ \\
\hline Elsewhere & $1996(87)$ \\
\hline Ethnicity & $294(13)$ \\
\hline White & \\
\hline & Other
\end{tabular}

\section{Colonoscopy outcomes}

\begin{tabular}{lc} 
Most advanced colorectal neoplasia & \\
Cancer & $6(0.3)$ \\
Advanced adenoma/serrated polyp & $176(8)$ \\
Non-advanced adenoma/serrated polyp & $540(24)$ \\
No adenoma/serrated polyp & $1513(66)$ \\
Polyp tissue not retrieved & $57(2)$ \\
\hline
\end{tabular}

CCSC, Colon Cancer Screening Centre. anti-inflammatory drugs, statins), screening behaviours, weight patterns, alcohol use, tobacco use, sociodemographic characteristics and anthropometric measurements. ${ }^{20}$

\section{Diet History Questionnaire}

Depending on the date of recruitment, participants completed either the Diet History Questionnaire (DHQ)-I (2008-2014) or DHQ-II (2014-2016). The DHQ is a selfadministered, past-year food and drink frequency questionnaire developed by the National Institutes of Health. ${ }^{21}$ Participants completed the Canadian version of the DHQ which is adapted for use in the Canadian population and incorporates standard vitamin and mineral enrichment of Canadian food products. ${ }^{21}{ }^{22}$ Key data from the DHQ includes derived variables related to the consumption of fibre, alcohol, red meat and processed meat. ${ }^{2122}$

\section{Long-form International Physical Activity Questionnaire}

The International Physical Activity questionnaire (IPAQ) measures physical activity levels and sedentary behaviour. ${ }^{23}$ Several physical activity types such as leisure time, household, occupational and transportation-related physical activity in the previous 7 days are collected. ${ }^{23}$ Frequency (days per week), duration (time per day) and intensity of each physical activity are also captured. IPAQ is available in short and long form, both of which are selfadministered and has acceptable validity in measuring physical activity levels and patterns among adults. ${ }^{23}$

\section{Medication,vitamin and nutritional supplement use}

Information on medications, vitamin and herbal and nutritional supplement use in the prior 30-day time period was collected from every participant at the time 
of enrolment. Information on each product includes the drug identification number or natural product number, dose, frequency and duration of use. Prior to collection of blood and urine, medications and supplements taken within the prior 24 hours is recorded.

\section{Blood and urine collection}

Blood and urine were collected during the same patient visit within 180 days prior to colonoscopy (46.6 days on average) and prior to starting any bowel preparation or diet changes. Blood samples are collected at the University of Calgary's Heritage Research Medical Clinic by qualified venipuncture technicians. Patients then provide a urine sample following blood collection. Data collected at time of biospecimen collection includes date and time of collection as well as time since the patient last ate or smoked. Biospecimens were processed within 2 hours of collection following the CCSC Biorepository Standard Operating Procedures (SOPs) for blood and urine collection, processing and storage ${ }^{24}{ }^{25}$ Further details on blood and urine collection of the CCSC Biorepository are described elsewhere. ${ }^{24}{ }^{25}$ Blood and urine samples are stored in $-80^{\circ} \mathrm{C}$ freezers and are monitored 24 hours a day with remote sensors.

\section{Colonoscopy report form and pathology results}

A colonoscopy report form was completed by the endoscopist after each procedure documenting the location, size and shape of each identified polyp or mass. A trained research assistant reviewed the final pathology report and assigned a histological type to each resected polyp. If a participant required more than one colonoscopy to clear the colon or polyps, a composite record was created. In some cases polyp samples from resected polyps may be obtained following Pathological review if sample volume permits.

\section{FINDINGS TO DATE}

From 2008 to 2016 , approximately 2292 participants have been enrolled into the CCSC Biorepository's Average Risk Cohort and provided biospecimens. Table 1 provides the demographic, health and lifestyle characteristics of the cohort as well as the most advanced colorectal neoplasia detected at colonoscopy. The participants are typical of the population of the city of Calgary (predominantly white and born in Canada). Other characteristics of the cohort included $44 \%$ reporting a body mass index of 25 -30 (overweight), $53 \%$ having never smoked tobacco and $13 \%$ having a family member with CRC.

\section{Patient and public involvement}

This research was done without patient involvement. Patients were not invited to comment on the study design and were not consulted to develop patient relevant outcomes or interpret the results. Patients were not invited to contribute to the writing or editing of this document for readability or accuracy.

\section{Strengths and limitations}

The richness of biological samples combined with comprehensive lifestyle and family history questionnaires is a unique and highly attractive population data source for researchers. The biorepository includes a variety of samples including serum, plasma, buffy coats, urine and normal colon tissues. Each of these different biospecimen are collected at the time of colonoscopy and are processed and stored with our validated SOPs. Sample processing metrics and questionnaires are collected for all biospecimen including time from collection, processing and freezing. Biospecimen samples coupled with participant data contained in the CCSC can inspire exploration into the development of new medications, treatments and research into the genetic causes and markers of CRC, along with other digestive diseases. The biorepository can also support innovative projects such as the evaluation and development of novel CRC screening tests, alternative methods to detect other digestive diseases, and the development of new treatments options. In the future, the biorepository can make it possible to study samples and make comparisons between people with and without certain conditions, such as colon cancer or polyps and different lifestyles to help understand the possible causes of disease and to develop new ways of detecting these conditions earlier. Finally, the biorepository can serve as an educational resource for scientists in the selection and collection of optimal biospecimen samples to support their research. ${ }^{26}$ As the cohort is continuing recruitment and additional data collection, future collaborative studies may be developed to answer specific investigator-initiated questions in this unique data collection framework.

The CCSC biorepository has several limitations. Currently, the main weakness is the relatively small population size of the cohort $(n=2292)$. The data collected through questionnaires only provides cross-sectional data, however, prospective data may be collected depending on individual study requirements. As time progresses the biorepository will begin to accrue repeat screening colonoscopies which will generate repeated screening measures data on a subset of the cohort. Like many biobank sources, variabilities in research using human tissue may be due to bias resulting from differences in the characteristics of the samples. ${ }^{26}$ Examples of potential sources of bias in biospecimen collection include: population differences (age, sex, race), comorbidities, medication use, time from collection or processing, time from processing to freezing and differences in length of storage time. ${ }^{26}$ To reduce potential biases, the CCSC adheres to specific SOPs for every step of the biospecimen and data collection process, handling, and storage. Lastly, as seen among other repositories, the CCSC may face common future challenges such as limited resources, storage capacity and oversight requirements to serve research operations. ${ }^{27}$ 


\section{Collaboration}

The CCSC biorepository is open for access to external researchers. Participants have consented to the use and sharing of their deidentified data and samples for future research purposes. Interested researchers can submit project proposals and request data or samples by contacting ccscresearch@ucalgary.ca. Ethics approval will be necessary prior to obtaining data. Biospecimens are available to researchers on a cost recovery basis. Researchers can visit the website at https://www.ucalgary. ca/colonscreening/biorepository or contact the biorepository at ccscresearch@ucalgary.ca to request samples. The website provides further details on questionnaires and the participant population. The CCSC Biospecimen Release Review Committee evaluates sample requests, and review applications for biospecimens after receipt of a completed application form and accompanying documents. All applications must have a current ethics approval certificate. The primary goal of the CCSC biorepository is to support research into colon cancer and other GI disorders. Applications will be assessed on how closely they meet this mandate.

\section{Further details}

In addition to the Average Risk Cohort, the CCSC Biorepository includes smaller cohorts, including (1) positive FIT/FOBT, (2) Personal History of neoplastic polyps and (3) Known CRC/large polyp prior to resection.

\author{
Author affiliations \\ ${ }^{1}$ Department of Medicine, Cumming School of Medicine, University of Calgary, \\ Calgary, Alberta, Canada \\ ${ }^{2}$ Cancer Epidemiology and Prevention Research, Alberta Health Services, Calgary, \\ Alberta, Canada \\ ${ }^{3}$ Department of Community Health Sciences, Cumming School of Medicine, \\ University of Calgary, Calgary, Alberta, Canada \\ ${ }^{4}$ Department of Oncology, University of Calgary Cumming School of Medicine, \\ Calgary, Alberta, Canada \\ ${ }^{5}$ Dalla Lana School of Public Health, University of Toronto, Toronto, Ontario, Canada
}

Acknowledgements We express our deepest gratitude to the participants who have selflessly taken the time and effort to provide their information and samples to support future research and innovation. In addition, we are grateful to the support and assistance of all of the nurses and endoscopists at the Forzani \& MacPhail Colon Cancer Screening Centre.

Contributors RJH and ST led study implementation. RJH, ST, DB and ML provided input on various aspects of study design. ST was responsible for the day-to-day project management. MDD, JE, ST, SC, CM, M-EM, EM, DB, and LR were actively involved in study planning and data collection. RJH, SJH, DB, AB and JP prepared the original draft of the manuscript. RJH, ST and ML conducted data analyses and put together tables and figures. All authors provided review of the manuscript and approved the final version.

Funding The CCSC Biorepository has been supported by funding from the Canadian Institutes of Health Research (Grant No. CIHR \#31863), the Alberta Cancer Foundation, the Alberta Heritage Foundation for Medical Research/Alberta Innovates - Health Solutions, Alberta Health Services and the University of Calgary (Grant No. 24850).

Competing interests None declared.

Patient consent for publication Not required.

Ethics approval Ethics approval for recruitment and data collection was initially received from the University of Calgary Conjoint Health Research Ethics Board (CHREB) and later transferred to the Health Research Ethics Board of Alberta Cancer Committee (ID HREBA.CC-16-0224).
Provenance and peer review Not commissioned; externally peer reviewed.

Data availability statement Data are available on reasonable request. Interested researchers can submit project proposals and request data or samples by contacting ccscresearch@ucalgary.ca. Ethics approval will be necessary prior to obtaining data. Additional information can be obtained through the CCSC website at https://www.ucalgary.ca/colonscreening/.

Supplemental material This content has been supplied by the author(s). It has not been vetted by BMJ Publishing Group Limited (BMJ) and may not have been peer-reviewed. Any opinions or recommendations discussed are solely those of the author(s) and are not endorsed by BMJ. BMJ disclaims all liability and responsibility arising from any reliance placed on the content. Where the content includes any translated material, BMJ does not warrant the accuracy and reliability of the translations (including but not limited to local regulations, clinical guidelines, terminology, drug names and drug dosages), and is not responsible for any error and/or omissions arising from translation and adaptation or otherwise.

Open access This is an open access article distributed in accordance with the Creative Commons Attribution Non Commercial (CC BY-NC 4.0) license, which permits others to distribute, remix, adapt, build upon this work non-commercially, and license their derivative works on different terms, provided the original work is properly cited, appropriate credit is given, any changes made indicated, and the use is non-commercial. See: http://creativecommons.org/licenses/by-nc/4.0/.

ORCID iDs

Robert J Hilsden http://orcid.org/0000-0003-1545-1093

Darren Brenner http://orcid.org/0000-0002-3027-290X

\section{REFERENCES}

1 Canadian Cancer Society. Canadian cancer society's advisory committee on cancer statistics: Canadian cancer statistics 2017 [PDF]. Canadian cancer society, statistics Canada, public health agency of Canada, provincial/territorial cancer registries. Available: www.cancer.ca/Canadian-Cancer-Statistics-2017-EN [Accessed 10 Jul 2018]

2 Safiri S, Sepanlou SG, Ikuta KS, et al. The global, regional, and national burden of colorectal cancer and its attributable risk factors in 195 countries and territories, 1990-2017: a systematic analysis for the global burden of disease study 2017. Lancet Gastroenterol Hepatol 2019;4:913-33.

3 Fearon ER, Vogelstein B. A genetic model for colorectal tumorigenesis. Cell 1990;61:759-67.

4 Jasperson KW, Tuohy TM, Neklason DW, et al. Hereditary and familial colon cancer. Gastroenterology 2010;138:2044-58.

$5 \mathrm{Ma}$ Y, Yang Y, Wang F, et al. Obesity and risk of colorectal cancer: a systematic review of prospective studies. PLoS One 2013;8:e53916.

6 Moghaddam AA, Woodward M, Huxley R. Obesity and risk of colorectal cancer: a meta-analysis of 31 studies with 70,000 events. Cancer Epidemiology Biomarkers \& Prevention 2007;16:2533-47.

7 Botteri E, lodice S, Bagnardi V, et al. Smoking and colorectal cancer. JAMA 2008;300:2765.

8 Wang $\mathrm{Y}$, Duan $\mathrm{H}$, Yang $\mathrm{H}$, et al. A pooled analysis of alcohol intake and colorectal cancer. Int J Clin Exp Med 2015;8:6878-89.

9 Sandhu MS, White IR, McPherson K. Systematic review of the prospective cohort studies on meat consumption and colorectal cancer risk: a meta-analytical approach. Cancer Epidemiol Biomarkers Prev 2001;10:439-46.

10 Chan DSM, Lau R, Aune D, et al. Red and processed meat and colorectal cancer incidence: meta-analysis of prospective studies. PLoS One 2011;6:e20456.

11 Hansen L, Skeie G, Landberg R, et al. Intake of dietary fiber, especially from cereal foods, is associated with lower incidence of colon cancer in the Helga cohort. Int. J. Cancer 2012;131:469-78.

12 Harriss DJ, Atkinson G, Batterham A, et al. Lifestyle factors and colorectal cancer risk (2): a systematic review and meta-analysis of associations with leisure-time physical activity. Colorectal Dis 2009;11:689-701.

13 Wolin KY, Yan Y, Colditz GA. Physical activity and risk of colon adenoma: a meta-analysis. Br J Cancer 2011;104:882-5.

14 Kondo Y, Issa J-PJ. Epigenetic changes in colorectal cancer. Cancer Metastasis Rev 2004;23:29-39.

15 Jia Y, Guo M. Epigenetic changes in colorectal cancer. Chin J Cancer 2013;32:21-30.

16 Haggar F, Boushey R. Colorectal cancer epidemiology: incidence, mortality, survival, and risk factors. Clin Colon Rectal Surg 2009;22:191-7. 
17 Canadian Partnership Against Cancer. Colorectal cancer screening in Canada: monitoring \& evaluation of quality indicators - results report, January 2013 - December 2014 [PDF]. Canadian partnership against cancer 2017. Available: http://www.cancerview.ca/preventi onandscreening/colorectalcancerscreeningpage/ [Accessed $10 \mathrm{Jul}$ 2018].

18 Canadian Task Force on Preventive Health Care. Recommendations on screening for colorectal cancer in primary care. Can Med Assoc J 2016;188:340-8.

19 Ebell MH, Thai TN, Royalty KJ. Cancer screening recommendations: an international comparison of high income countries. Public Health Rev 2018;39.

20 Hawkins M, Gill SD, Batterham R, et al. The health literacy questionnaire (HLQ) at the patient-clinician interface: a qualitative study of what patients and clinicians mean by their HLQ scores [Internet]. BMC Health Serv Res, 2017. Available: https:// bmchealthservres.biomedcentral.com/articles/10.1186/s12913-0172254-8 [Accessed 10 Jul 2018]

21 National Cancer Institute. Diet history questionnaire II (DHQ II) for U.S. \& Canada. [Website]. Epidemiology and genomics research program, 2007. Available: http://epi.grants.cancer.gov/DHQ/ [Accessed 10 Jul 2018].
22 Csizmadi I, Kahle L, Ullman R, et al. Adaptation and evaluation of the National cancer Institute's diet history questionnaire and nutrient database for Canadian populations. Public Health Nutr 2007;10:88-96.

23 Craig CL, Marshall AL, Sjöström M, et al. International physical activity questionnaire: 12 -country reliability and validity. Med Sci Sports Exerc 2003;35:1381-95.

24 Forzani \& MacPhail Colon Cancer Screening Centre Biorepository. Blood collection, processing \& storage standard operating procedure [Internet]. SOPs | Colon cancer screening centre | University of Calgary. Available: dx.doi.org/10.17504/protocols.io.wiifcce [Accessed 22 Jan 2019].

25 Forzani \& MacPhail Colon Cancer Screening Centre Biorepository. Urine collection, processing \& storage standard operating procedure [Internet]. SOPs | Colon cancer screening centre | University of Calgary. Available: dx.doi.org/10.17504/protocols.io.w94fh8w [Accessed 22 Jan 2019].

26 Grizzle WE, Gunter EW, Sexton KC, et al. Quality management of Biorepositories. Biopreserv Biobank 2015;13:183-94.

27 Betsou F, Rimm DL, Watson $\mathrm{PH}$, et al. What are the biggest challenges and opportunities for Biorepositories in the next three to five years? Biopreserv Biobank 2010;8:81-8. 